\title{
Multimodal Imaging of Cotton Wool Spots in Branch Retinal Vein Occlusion
}

\author{
Marion R. Munk ${ }^{a, b, e}$ Gerlinde Matt ${ }^{a, b, f}$ Magdalena Baratsits ${ }^{a, b}$ \\ Roman Dunavoelgyi ${ }^{a, b}$ Wolfgang Huf ${ }^{c}$ Alessio Montuoro ${ }^{a, d}$ Wolf Buehl ${ }^{a, b}$ \\ Ursula Schmidt-Erfurth ${ }^{a, b}$ Stefan Sacu ${ }^{a, b}$ on behalf of the Macula Study Group \\ ${ }^{a}$ Department of Ophthalmology, ${ }^{\mathrm{b}}$ Medical University of Vienna, ${ }^{\mathrm{C} C e n t e r}$ for Medical Physics and Biomedical \\ Engineering and d Vienna Reading Center, Medical University of Vienna, Vienna, Austria; ${ }^{e}$ Department of \\ Ophthalmology, Inselspital, University Hospital Bern, Bern, and fDepartment of Ophthalmology, University Hospital \\ Zurich, Zurich, Switzerland
}

\author{
Key Words \\ Ischemia · Cotton wool spots - Cystoid bodies · Argon \\ laser treatment $\cdot$ Retinal vein occlusion - Branch retinal \\ vein occlusion · Hypoperfusion - Optical coherence \\ tomography · Fluorescein angiography · Axoplasmic \\ debris · Multimodal imaging · Acute macular \\ neuroretinopathy
}

\begin{abstract}
Purpose: To describe and follow cotton wool spots (CWS) in branch retinal vein occlusion (BRVO) using multimodal imaging. Methods: In this prospective cohort study including 24 patients with new-onset BRVO, CWS were described and analyzed in color fundus photography (CF), spectral domain optical coherence tomography (SD-OCT), infrared (IR) and fluorescein angiography (FA) every 3 months for 3 years. The CWS area on SD-OCT and CF was evaluated using OCT-ToolKit software: CWS were marked in each single OCT B-scan and the software calculated the area by interpolation. $\boldsymbol{R e}$ sults: 29 central CWS lesions were found. $100 \%$ of these CWS were visible on SD-OCT, $100 \%$ on FA and $86.2 \%$ on IR imaging, but only $65.5 \%$ on CF imaging. CWS were visible for $12.4 \pm 7.5$ months on SD-OCT, for $4.4 \pm 3$ months and $4.3 \pm$ 3.4 months on CF and on IR, respectively, and for $17.5 \pm 7.1$ months on FA. The evaluated CWS area on SD-OCT was larg-
\end{abstract}

er than on CF $\left(0.26 \pm 0.17 \mathrm{~mm}^{2}\right.$ vs. $\left.0.13 \pm 0.1 \mathrm{~mm}^{2}, \mathrm{p}<0.0001\right)$. The CWS area on SD-OCT and surrounding pathology such as intraretinal cysts, avascular zones and intraretinal hemorrhage were predictive for how long CWS remained visible $\left(r^{2}=0.497, p<0.002\right)$. Conclusions: The lifetime and presentation of CWS in BRVO seem comparable to other diseases. SD-OCT shows a higher sensitivity for detecting CWS compared to CF. The duration of visibility of CWS varies among different image modalities and depends on the surrounding pathology and the CWS size.

(c) 2015 S. Karger AG, Basel

\section{Introduction}

Cotton wool spots (CWS) are not specific for a disease, but rather a sign of vascular damage [1]. Ischemia due to vasoconstriction or occlusive events as well as mechanical pressure affecting the superficial capillary network located in the retinal nerve fiber layer (RNFL) may induce CWS. In turn, ischemic events of the intermediate and deep capillary network may induce paracentral middle maculopathy and acute macular neuroretinopathy. Histologically, CWS consist of accumulated cystoid bodies, which represent the terminal swelling of a disrupted ganglion cell axon with accumulated axonal debris $[2,3]$. 
CWS reveal a characteristic presentation on each image device. On color fundus photographs (CF) or by ophthalmoscopy, CWS appear as fluffy whitish lesions with frayed edges [1]. In fluorescein angiography (FA), $90 \%$ of the lesions appear hyperfluorescent and the remaining $10 \%$ appear hypofluorescent in the early phase and become hyperfluorescent in the late phase [4]. In optical coherence tomography (OCT), CWS appear as hyperreflective lesions in the retinal RNFL [5]. On average, CWS disappear within 7-12 weeks from ophthalmoscopic view, although they have been found to be still visible on OCT after vanishing from $\mathrm{CF}$ images [5-7]. After resolution on OCT, CWS often leave ganglion cell layer, RNFL and inner nuclear layer thinning, and functionally CWS resolution is associated with persistent relative scotomas $[8,9]$.

CWS have been methodically studied in HIV, diabetic macular edema, central retinal vein occlusion and hypertension, but so far not in branch retinal vein occlusion (BRVO) [8-13]. The aim of this study was to assess and describe CWS in BRVO in a longitudinal setting over 3 years using multimodal imaging.

\section{Methods}

\section{Patient Selection and Setting}

30 eyes of 30 patients with new-onset BRVO, who were recruited for a prospective nonrandomized study at the Department of Ophthalmology, Medical University Vienna, were evaluated [14]. The study adhered to the tenets of the Declaration of Helsinki, was approved by the Ethics Committee of the Medical University of Vienna and all patients gave informed consent. Inclusion criteria were the confirmed diagnosis of treatment-naïve acute BRVO with a decrease of visual acuity and a central retinal thickness $>300 \mu \mathrm{m}$. Patients received one intravitreal ranibizumab (Lucentis; Novartis, Basel, Switzerland) injection at baseline, followed by an 'as needed' regimen (treatment was repeated when central retinal thickness increased $>50 \mu \mathrm{m}$ compared with the previous visit or when BCVA decreased $>5$ letters). Study visits in the first year were scheduled on a monthly basis followed by 3 -month intervals for the next 2 years. Segmental argon laser coagulation was performed in patients with an avascular zone of more than 5 papillary diameters and recurrent macular edema on FA, and after sufficient clearance of intraretinal hemorrhage. Patients were followed up for 3 years (for further details regarding study design see 'Ranibizumab in patients with branch retinal vein occlusions', ClinicalTrials.gov Identifier: NCT01027481, https://clinicaltrials. gov/ct2/show/NCT01027481).

Patients who had had BRVO longer than 8 weeks before the start of the study were not included to assure a homogeneous group of CWS with recent onset. CF, FA, infrared (IR) and spectral domain (SD)-OCT imaging were performed and evaluated at baseline and thereafter at 3-month intervals for 3 years. The number of ranibizumab injections and argon laser coagulation laser treatments were recorded.

Multimodal Imaging of CWS in BRVO
Image Acquisition and Evaluation

Spectralis SD-OCT (Heidelberg Engineering, Heidelberg, Germany) volume scans and IR images (37 high-resolution scans, distance between scans $124 \mu \mathrm{m}, 30$ ART frames, $20 \times 15$, Heidelberg Engineering, Software 1.7.0.0) were evaluated at baseline and every 3 months thereafter. Scans at exactly the same retinal location were generated at each visit using the follow-up function. FA was performed with a Heidelberg retina angiograph (HRA 2, Heidelberg Engineering) and 2- and 10-min images were chosen for evaluation. CF photographs were taken with the Zeiss FF450 Fundus Camera (Carl Zeiss Meditec).

A software program (OCT-Tool-Kit) developed at the Vienna Reading Center was used to compare, measure and describe the CWS in the different image modalities $[15,16]$. SD-OCT and corresponding IR pictures were loaded in XML format, and FA and CF pictures were loaded in BMP format. Three easily detectable points in the IR picture and the corresponding points in the FA or CF picture were chosen with the OCT-Tool-Kit software. Then, CF and FA pictures were superimposed exactly over the IR images. The XML format included additional image information (e.g. pixel/ $\mu \mathrm{m}$ ) provided by the OCT manufacturer, which enables the software to compare the BMP pictures (thus CF and FA images) directly and 1:1 to the SD-OCT-images, irrespective of their individual original magnifications. This allows a direct 1:1 comparison of appearance and size. The CWS were then marked in every single OCT B-scan. The software projected the marked areas in the en-face IR picture, calculating the CWS area by interpolation. Hereby, the manually marked CWS areas in the B-scan and the mean distance between the B-scans $(0.012 \mathrm{~mm}$ in the 37-line scan) together with the XML file software calibration were used to calculate the area of each CWS. The area in CF photographs was evaluated in the same way by uploading BMP images and manually marking affected areas in every B-scan.

Due to the exact overlay, a qualitative and quantitative pointto-point comparison of the size, expanse, morphology and appearance of CWS in SD-OCT and the corresponding imaging devices was possible. A CWS was defined as present in SD-OCT when a homogenous plaque of intensive hyperreflectivity at the neuronal layer and underneath was associated with a focal epiretinal elevation in at least one B-scan [5]. The subsequent thinning of the RNFL, ganglion cell layer and inner nuclear layer was not graded as presence of a CWS [9]. A CWS was graded as present in CF images when a circumscribed, whitish, fluffy lesion with frayed edges was seen [1]. A CWS was graded as present on IR images when a well-demarcated whitish hyperreflective roundish lesion corresponding to the hyperreflective RNFL thickening in SD-OCT or the fluffy whitish lesion on CF images was present. A CWS was graded as present on FA when a well-demarcated hypo/hyperfluorescent lesion corresponding to the presence of a CWS in SD-OCT and/or CF images was present (fig. 1).

\section{Statistical Analyses}

Statistical analyses were performed using SPSS 21 (SPSS Inc., Chicago, Ill., USA) software. Data were evaluated descriptively and numeric values were indicated as the mean \pm SD. Differences in terms of size were evaluated with a paired $t$ test. Factors associated with the duration of visibility of CWS were analyzed using a multivariate regression analysis. $\mathrm{p} \leq 0.05$ was deemed statistically significant. 

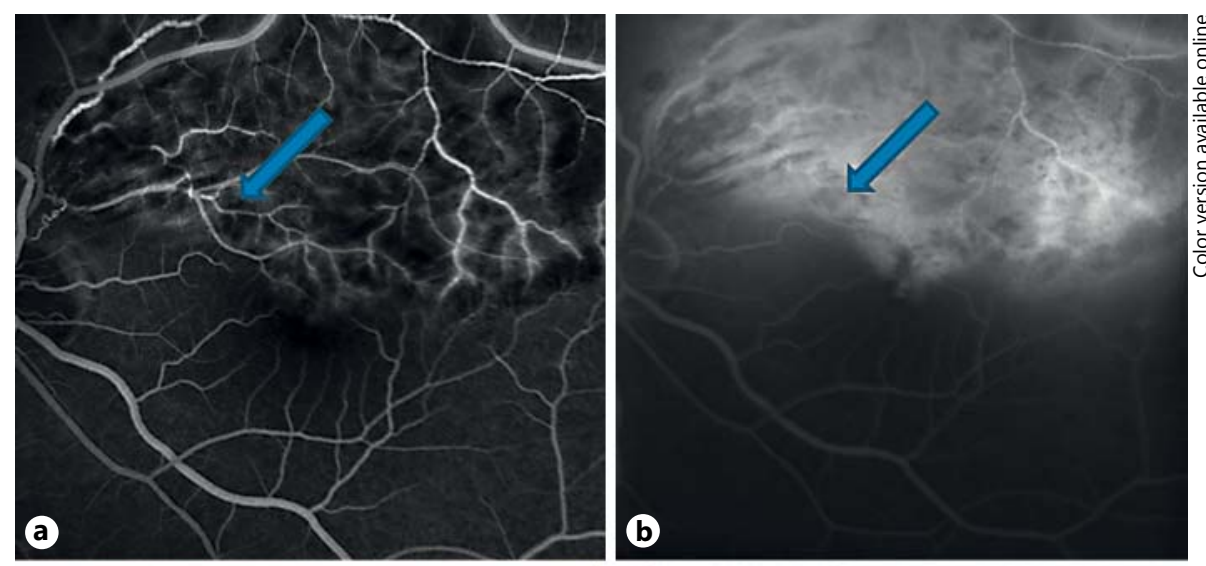

Fig. 1. CWS at baseline in FA in a 2-min phase (a) and in a 10-min phase (b), CF (c), IR (d) and SD-OCT (e). In SD-OCT the indicated CWS (arrow) presented as a homogenous plaque of intensive hyperreflectivity in the neuronal layer. In CF the CWS appeared as a circumscribed, whitish, fluffy lesion with frayed edges. In IR, the CWS presented as a well-demarcated whitish hyperreflective roundish lesion, corresponding to the hyperreflective nerve fiber layer thickening in SD-OCT or the fluffy whitish lesion in CF. In FA, the corresponding CWS area was either hypofluorescent, hyperfluorescent, or hypofluorescent and became hyperfluorescent in the late phase.
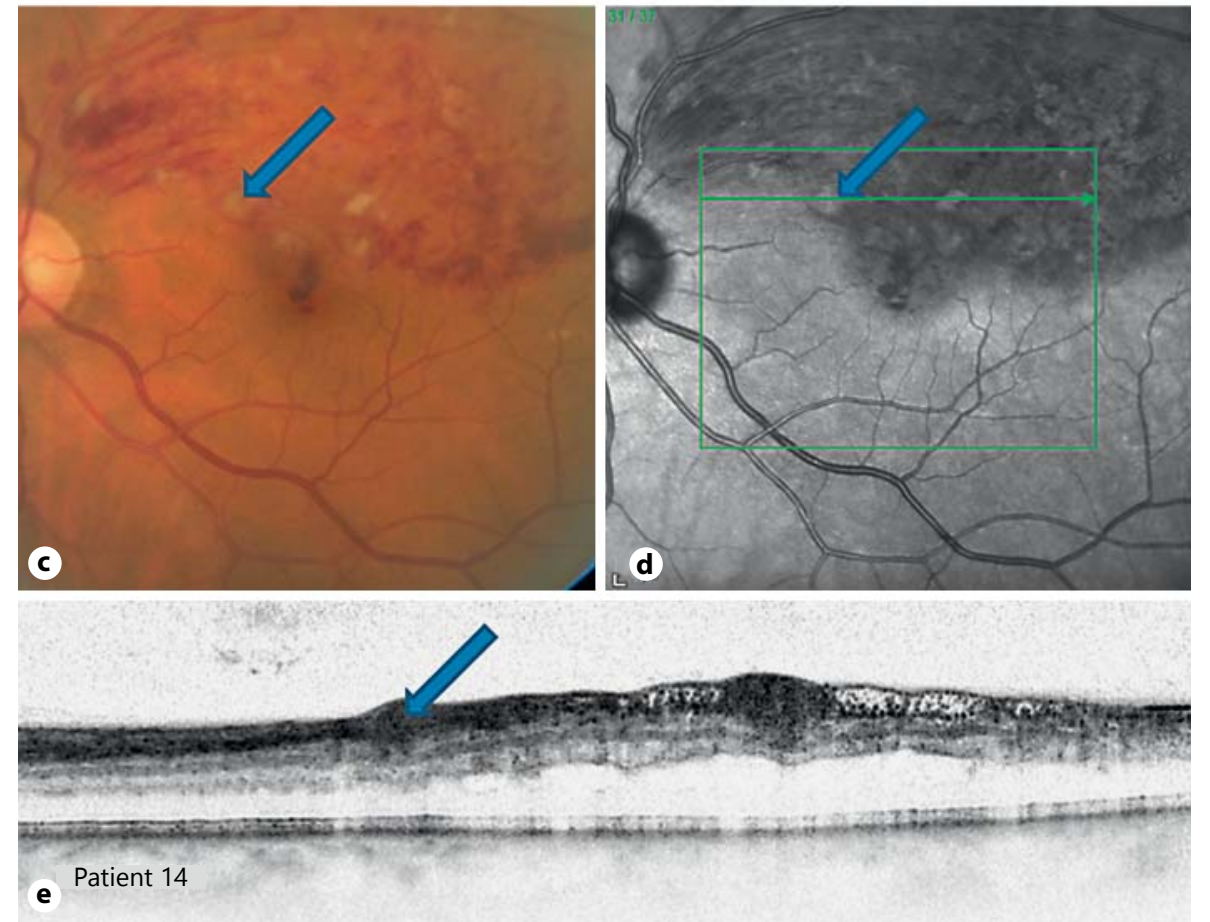

\section{Results}

\section{Demographic Characteristics}

Out of the 30 patients, 24 patients ( 12 men, 12 women, mean age: $66 \pm 11$ years, 4 inferotemporal and 20 superotemporal BRVOs) presented with BRVO with an onset $\leq 8$ weeks before the screening visit and were included in the study for further evaluation. Out of these 24 eyes, 18 eyes $(75 \%)$ showed one or more CWS within the raster scan area (mean: $1.8 \pm 1.3$ CWS, range: $1-5$ ) per eye. Overall, 29 CWS lesions within the SD-OCT scan area were found and analyzed at baseline. During the 3-year observational period, 3 additional CWS appeared in 3 different patients. A representative example of the presentation of a
CWS in respective imaging devices can be found in figure 1. Figure 2 shows the corresponding marked areas. The average number of ranibizumab injections per patient was $4.5 \pm 3.7$ (range: $1-13$ ) over the 3 -year period. Out of the 24 patients, 6 ( 5 presenting with CWS, 1 not) received segmental argon laser coagulation laser treatment (mean: $1.7 \pm 0.7$, range: $1-3$ ) during the observational period.

\section{Morphologic and Angiographic Appearance of CWS}

All 29 CWS (100\%) were visible on SD-OCT, but only 19 (65.5\%) were visible on the CF images at baseline. 25 (86.2\%) of the CWS lesions showed corresponding changes on the IR image. All 29 (100\%) CWS lesions showed corresponding fluorescent changes on FA: 9 (31\%) 
Fig. 2. The different marked areas of the CWS in SD-OCT (corresponding to fig. 1), FA and CF evaluated by the OCT-Tool Kit software. CWS were marked in each OCT B-scan (a), and the marked area was then projected en-face on the IR picture (b) and the affected area was automatically calculated by interpolation. The CWS (arrow) was $0.14 \mathrm{~mm}^{2}$ in SD-OCT (c) $0.09 \mathrm{~mm}^{2}$ in CF (arrow) (d) and the corresponding CWS area in FA revealed $0.17 \mathrm{~mm}^{2}$.
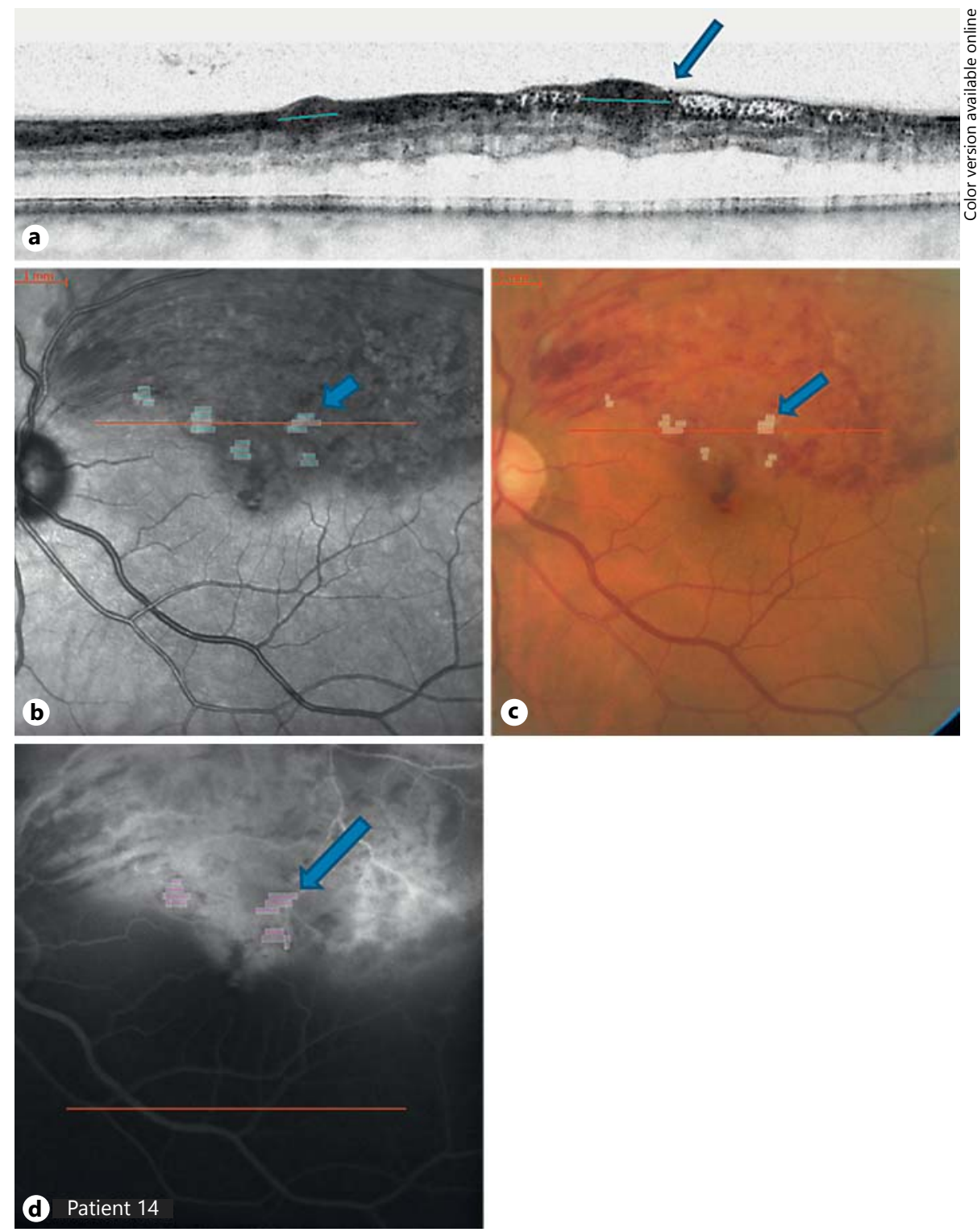

showed hypofluorescent lesions, 4 (13.8\%) hyperfluorescent lesions, and the majority, 16 (55.2\%), hypofluorescent changes at $2 \mathrm{~min}$ and hyperfluorescent changes at $10 \mathrm{~min}$. At baseline, 27 (92.1\%) CWS were surrounded by blood visible on CF images, $23(78.3 \%)$ by intraretinal cysts visible on OCT, 2 (6.9\%) by subretinal fluid and 1 by an avascular zone visible on FA.

CWS on FA could change their fluorescent behavior on FA as the fluorescence seemed to be associated with the surrounding pathology: CWS in the near vicinity of intraretinal cysts were mainly hyperfluorescent (88\%), CWS surrounded by intraretinal hemorrhage were either hypofluorescent (41\%) or hypofluorescent in the early phase and hyperfluorescent in late phase (44\%), and CWS within an avascular zone presented mostly (83\%) as hypofluorescent on FA.

\section{Size and Duration of Visibility}

The mean area of CWS for each imaging method is shown in table 1. The mean area of a CWS in SD-OCT was larger than on the CF image (paired t test: $0.26 \pm 0.17$ $\mathrm{mm}^{2}$ vs. $\left.0.13 \pm 0.1, \mathrm{p} \leq 0.0001\right)$. The CWS area evaluated by the OCT-Tool-Kit software on SD-OCT (but not on $\mathrm{CF}$ ), and surrounding pathology such as intraretinal cysts, avascular zones and intraretinal hemorrhage were predictive for how long a CWS remained visible $\left(\mathrm{r}^{2}=\right.$ 
Table 1. Mean calculated area of CWS in BVRO in SD-OCT, CF and FA

\begin{tabular}{llll}
\hline $\begin{array}{l}\text { Visits, } \\
\text { months }\end{array}$ & SD-OCT, $\mu \mathrm{m}^{2}$ & $\mathrm{CF}, \mu \mathrm{m}^{2}$ & $\mathrm{FA}, \mu \mathrm{m}^{2}$ \\
\hline Baseline & $0.22 \pm 0.16$ & $0.12 \pm 0.09$ & $0.27 \pm 0.29$ \\
3 & $0.19 \pm 0.13$ & $0.08 \pm 0.05$ & $0.25 \pm 0.21$ \\
6 & $0.18 \pm 1.9$ & $0.17 \pm 0.12$ & $0.3 \pm 0.22$ \\
9 & $0.09 \pm 0.07$ & 0.06 & $0.12 \pm 0.1$ \\
12 & $0.08 \pm 0.03$ & 0.03 & $0.12 \pm 0.7$ \\
15 & $0.08 \pm 0.07$ & 0.03 & $0.29 \pm 0.47$ \\
18 & $0.09 \pm 0.06$ & n.a. & n.a. \\
21 & $0.06 \pm 0.04$ & n.a. & n.a. \\
24 & $0.06 \pm 0.04$ & n.a. & n.a. \\
27 & $0.1 \pm 0.06$ & n.a. & n.a. \\
30 & 0.1 & n.a. & n.a. \\
33 & n.a. & n.a. & n.a. \\
36 & n.a. & n.a. & n.a. \\
\hline
\end{tabular}

Values are given as means \pm SD. Area of CWS on FA was evaluated until month 15. After that, the margins of CWS on FA were too blurry to allow accurate area evaluation.

0.497, $\mathrm{p}<0.002)$. Large CWS surrounded by intraretinal hemorrhage were visible longer than smaller CWS in the near vicinity of intraretinal cysts and avascular zones.

CWS were visible for $12.4 \pm 7.5$ months (range: $1-30$ ) on SD-OCT, but only for $4.4 \pm 3.2$ months (range: $1-12$ ) on CF images. Representative examples can be found in figures 3, 4 and 5. Corresponding CWS lesions on IR images were visible for $4.3 \pm 3.4$ months (range: $1-12$ ) while corresponding fluorescent changes on FA were visible longer than the CWS itself on SD-OCT (mean: $17.5 \pm 7.1$ months, range: 3-27). However, after the 15-month follow-up, the margins of the CWS on FA became very indistinct and the lesions were only discernable from their surroundings with the use of an overlay.

\section{Discussion}

To the best of our knowledge this is the first study to evaluate the appearance of CWS in a very homogeneous group of patients with BRVO using four different imaging modalities in a longitudinal manner. Although CWS have been described in different diseases, including CRVO, only a few case reports have addressed the appearance and behavior of CWSs in BRVO so far $[13,17]$. The current study reveals that SD-OCT aims to be a sensitive tool to detect small CWS even when they are invisible on $\mathrm{CF}$, and it demonstrates that the duration of visi- bility of CWS seems to differ dependent on image modality and appears to be associated with the size of a CWS and with the surrounding pathology.

Currently, the main clinical image modality to observe CWS is CF photography. This study, however, shows that only $65.5 \%$ of all examined CWS were detectable on CF, whereas all CWS were visible on SD-OCT and FA. Further, the area of a CWS was larger on SD-OCT compared to CF. Previous studies have already demonstrated that CWS are visible longer on OCT than by ophthalmoscopy $[2,9]$. The higher prevalence of CWS on SD-OCT may therefore be a result of the longer duration of visibility. It may also be possible that a CWS has to reach a certain size to be clearly visible by ophthalmoscopy.

Many previous studies have evaluated the mean lifetime' of CWS in funduscopy in different kinds of retinal diseases. These studies showed they were visible anywhere from 4 weeks to 17.2 months depending on underlying disease, patient's age and medication $[12,13,18]$. Besides these factors, our analysis revealed that the area of CWS as well as the surrounding pathology such as intraretinal cysts, intraretinal hemorrhage and avascular zones seem to be factors associated with the duration of visibility. On average, CWS were visible on CF images for 4.4 months in our study, which is right in the middle of previous findings. However, CWS were visible three times longer in SD-OCT than on CF images and IR, with a mean of 12 months. Previous studies have already shown that CWS are visible longer on OCT than on CF images and that the hyperreflectivity of the CWS lesions decreases gradually on OCT $[5,9]$. Similar to CF, the presence of CWS was shorter on IR than on OCT.

CWS result from ischemic events at the level of the superficial capillary network and present as hyporeflective lesions on IR and as localized hyperreflectivity and thickening of the RNFL on OCT. This presentation corresponds to the appearance of acute macular neuroretinopathy and paracentral middle maculopathy lesions on IR and SD-OCT, which are thought to be induced by vasoocclusive events at the level of the intermediate and deep retinal capillary network, and are therefore sometimes reported as 'deep CWS' [19-21]. However, unlike the current finding in CWS that the hyperreflectivity on OCT was visible longer than the hyporeflectivity on IR, acute macular neuroretinopathy and paracentral middle maculopathy lesions were reported to diminish simultaneously in respective imaging devices [19-22]. This discrepancy may be due to the fact that the CWS we examined were mainly found near other pathologies such as cysts, avascular zones or intraretinal hemorrhage, which may 
Fig. 3. Representative CWS in CF (a-d) and SD-OCT (e-h) during follow-up. a Baseline (size: $0.09 \mathrm{~mm}^{2}$ ). b 3 months after initial presentation, the area is even bigger compared to baseline (area: $0.15 \mathrm{~mm}^{2}$ ). c 6 months after initial presentation, CWS is no longer visible in CF. d 24 months after initial presentation. e Axoplasmic debris in the RNFL at baseline (area: $0.14 \mathrm{~mm}^{2}$ ). f Hyperreflective axoplasmic debris 3 months after initial presentation (area: $\left.0.28 \mathrm{~mm}^{2}\right)$. g 6 months after initial presentation, homogenous hyperreflective plaque is still visible in SD-OCT (area: $0.09 \mathrm{~mm}^{2}$ ). h 24 months after initial presentation, hyperreflective plaque has given way to inner retinal layer atrophy.

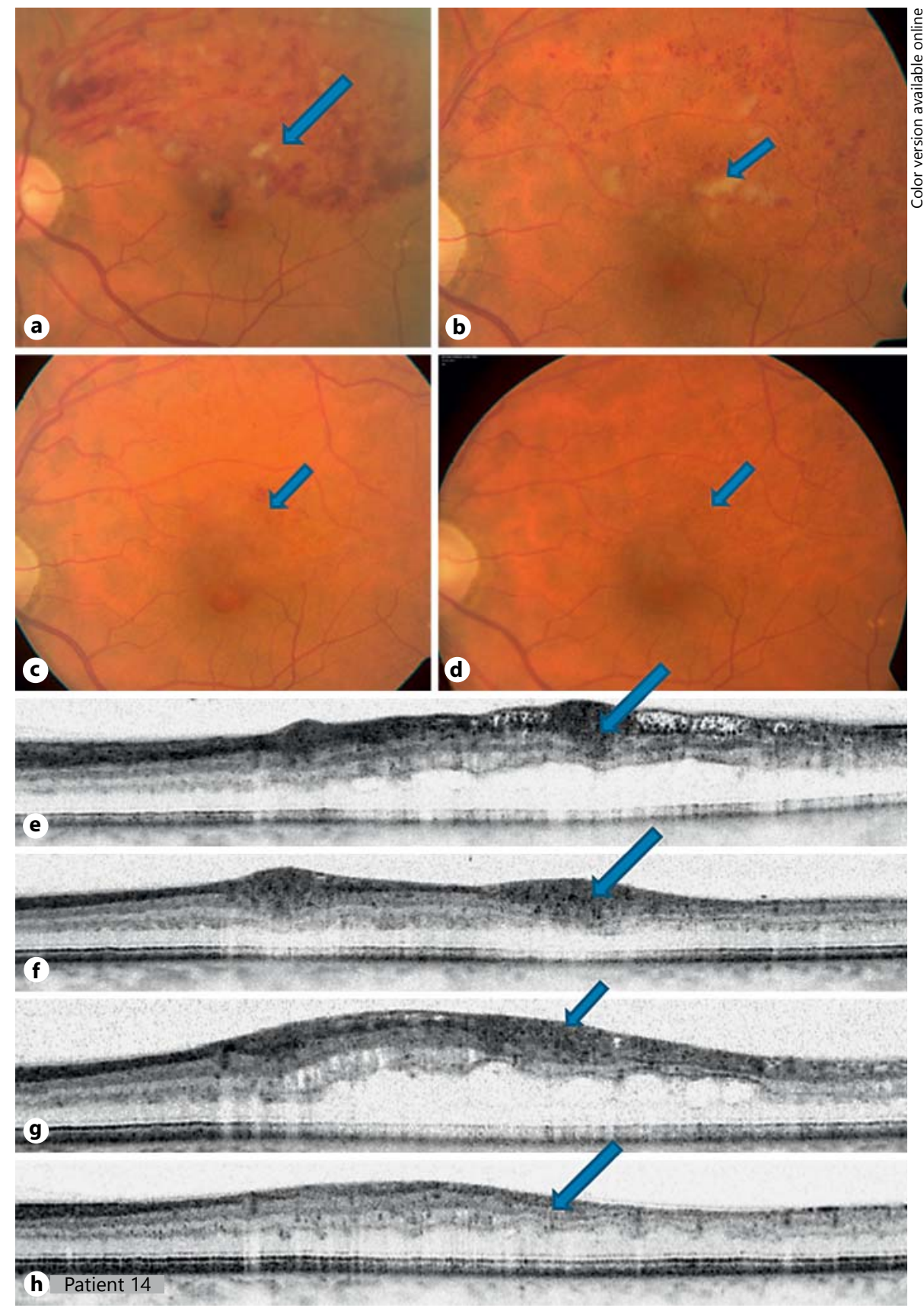

aggravate the evaluation of the CWS in different image devices and alter their presentation and behavior.

Interestingly, CWS remained visible for $17.5 \pm 7.1$ months on FA, longer than on SD-OCT. Previous studies have described superficial capillary nonperfusion with vascular leakage and staining in the late phase to correspond to the CWS on FA in the acute phase [21]. In the chronic phase, CWS were mainly described as in- visible on FA or detectable as an indistinct hypofluorescent area, visible due to the leakage from the surrounding perfused capillaries [21]. In our study, CWS were detectable on FA longer than on SD-OCT. It has to be taken into account, however, that hyperreflectivity and thickening of the RNFL, but not the ensuing localized atrophy of the RNFL, was graded as CWS on SD-OCT and that during follow-up the margins of the CWS on 
Fig. 4. Another example of the presentation of a CWS in CF (a-c) and SD-OCT (d-f). a Baseline (area: $0.22 \mathrm{~mm}^{2}$ ). b 6 months after initial presentation, the CWS has faded but is still visible (area: $0.1 \mathrm{~mm}^{2}$ ). c 12 months after initial presentation, the CWS is no longer visible in CF. $\mathbf{d}$ Axoplasmic debris in the RNFL at baseline (area: 0.3 $\mathrm{mm}^{2}$ ). e After 6 months: besides the atrophy of the inner retinal layers, a hyperreflective sign is still visible (area: $0.13 \mathrm{~mm}^{2}$ ). f After 12 months: concordant to the CFimages, the hyperreflective sign faded and the atrophy of the inner retinal layers remains.
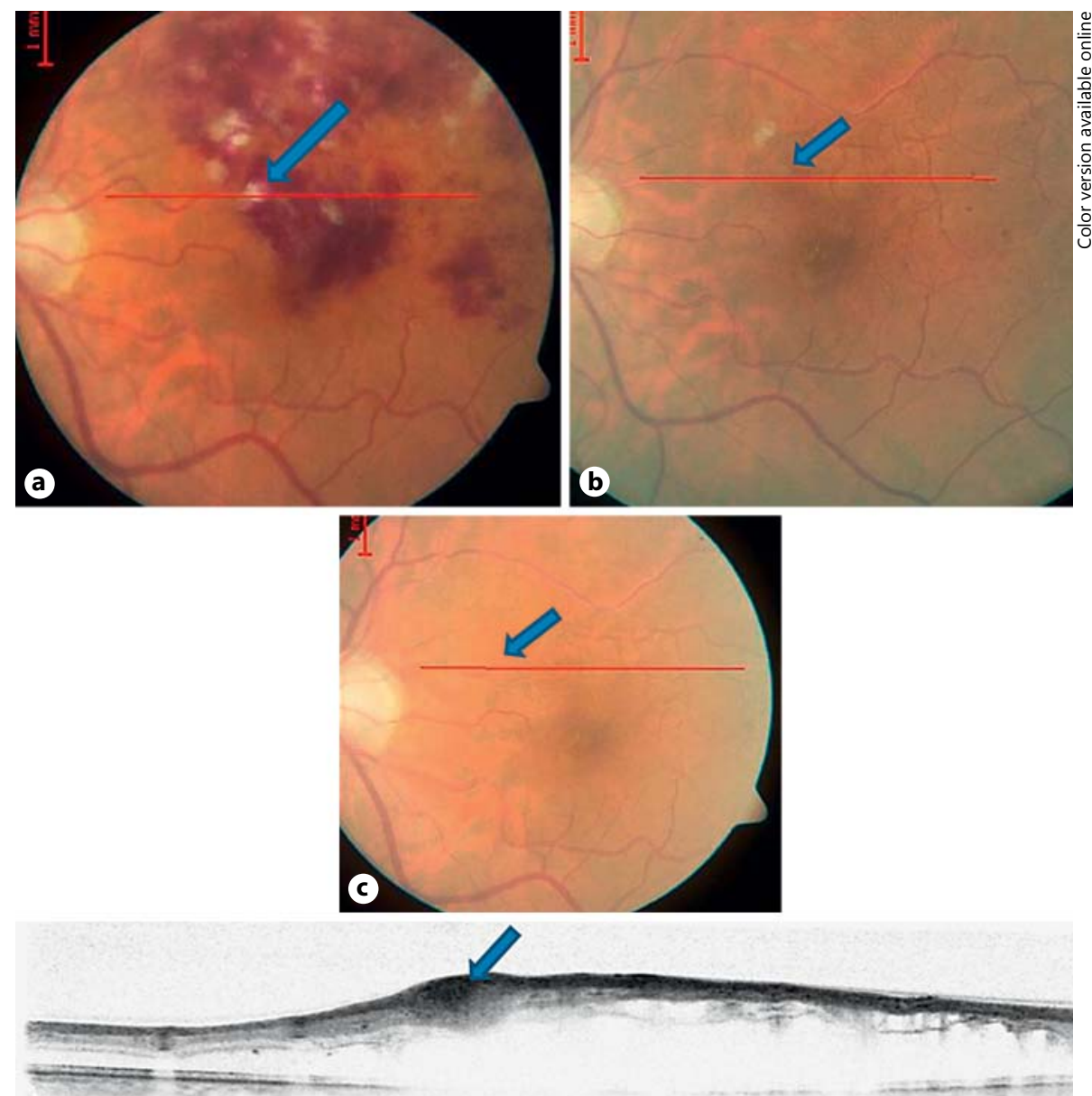

d

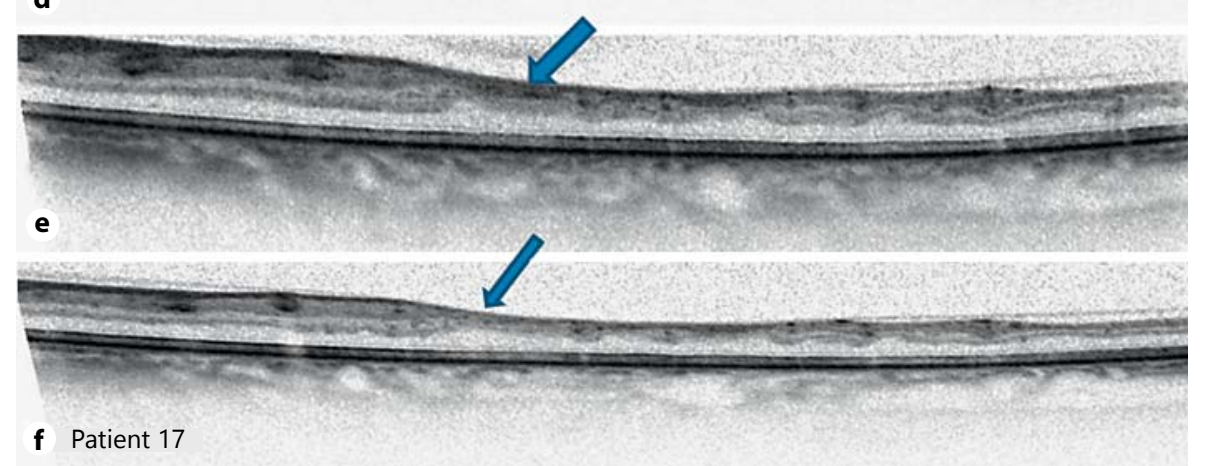

FA became very indistinct and the lesion was only discernable from the surroundings when an overlay was used. It seems reasonable that the affected area remains distinguishable for a long period of time on FA as it resembles an area of capillary nonperfusion which remains nonperfused [21].

Indeed, the surrounding pathology together with the size of a CWS has an impact on the duration of visibility.
Large CWS surrounded by intraretinal hemorrhage were visible longer than smaller CWS close to intraretinal cysts or an ischemic zone. The surrounding pathology not only affected the duration of visibility, but also influenced the fluorescent behavior of a CWS. In previous reports, CWS in FA were mainly described as hyperfluorescent lesions with late staining and leakage around their margins $[1,10$, $13,23]$. Lesions that were hypofluorescent in the early 
Fig. 5. Another example of the presentation of a CWS in CF (a-c) and SD-OCT (d-f). a Baseline (area: $0.22 \mathrm{~mm}^{2}$ ). b 6 months after initial presentation: the CWS area is even bigger compared to baseline (area: $0.28 \mathrm{~mm}^{2}$ ). c 12 months after initial presentation: CWS is no longer visible in CF. d Axoplasmic debris in RNFL at baseline (area: $0.51 \mathrm{~mm}^{2}$ ). e 6 months: the CWS area has increased in size (area: $0.79 \mathrm{~mm}^{2}$ ). $\mathbf{f} 18$ months: a hyperreflective sign is still visible (area: $0.17 \mathrm{~mm}^{2}$ ).
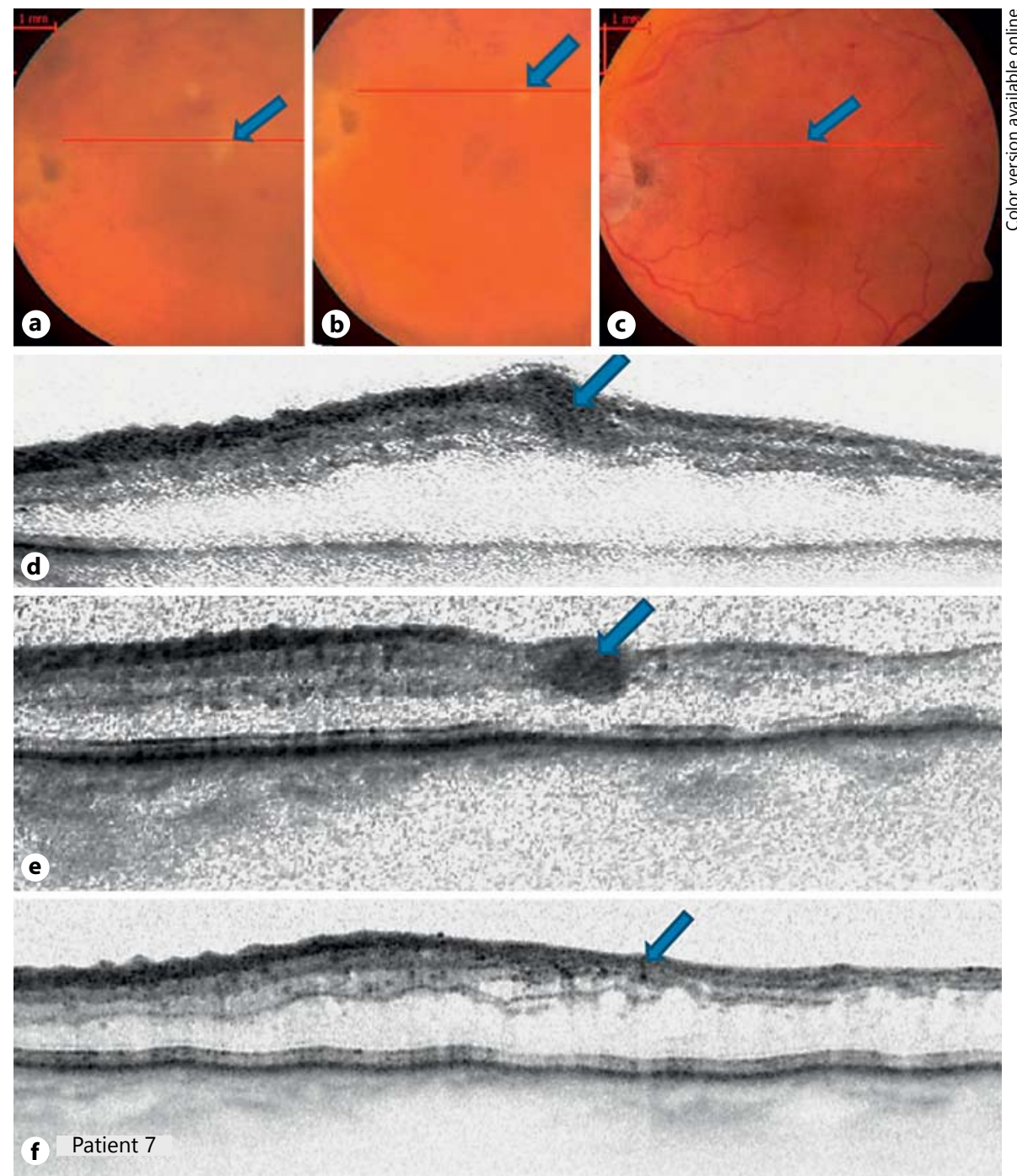

phase but became hyperfluorescent in the late phase were suggested to be a sign of ischemia and were observed in a study with experimental retinal ischemia [4]. In the current study, CWS were either hyperfluorescent or hypofluorescent in the early and late phases or hypofluorescent in the early phase and hyperfluorescent in the late phase. Their fluorescent behavior changed over time and was associated with the surrounding pathology: CWS surrounded by an avascular zone or by intraretinal hemorrhage presented as hypofluorescent and either remained hypofluorescent or became hyperfluorescent in the late phase due to staining and leakage. CWS surrounded by intraretinal cysts and edema, however, mainly presented as hyperfluorescent in the early and late phases. The discrepancies between the presentation of CWS on FA in experimental retinal ischemia and in
BRVO in the current study may be due to different findings in the area surrounding a CWS or due to different underlying pathologies. However, both have in common that hypofluorescent rather than pure hyperfluorescent CWS lesions seem to be a sign of hypoperfusion and ischemia. Intraretinal hemorrhage and focal ischemia were pathologies often described in close vicinity to CWS [23, 24 ], and since intraretinal hemorrhage is associated with local ischemia and often results in inner retinal atrophy, intraretinal hemorrhage could therefore be a concrete sign of the presence and the development of an avascular zone with similar results as an avascular zone in a hypofluorescent lesion.

In summary, this study indicates that the 'lifetime' of CWS in BRVO seems comparable to other diseases and that the duration of visibility of CWS varies among dif- 
ferent image modalities and depends on the surrounding pathology and the size of the CWS. Overall, SD-OCT seems to be a precise tool to detect, measure and observe central CWS, and it may be more sensitive in the detection of a CWS than CF.

\section{Disclosure Statement}

This study was supported by the Österreichische Nationalbank (Austrian National Bank) Jubiläumsfonds (Grant Nr. 12745). Ursula Schmidt-Erfurth received consultancy fees from Novartis. The remaining authors have no competing interests to disclose.

\section{References}

1 Schmidt D: The mystery of cotton-wool spots - a review of recent and historical descriptions. Eur J Med Res 2008;13:231-366.

2 Kozak I, et al: In vivo histology of cotton-wool spots using high-resolution optical coherence tomography. Am J Ophthalmol 2006;141: 748-750.

3 McLeod D: Why cotton wool spots should not be regarded as retinal nerve fibre layer infarcts. Br J Ophthalmol 2005;89:229237.

4 Tso MO, et al: Microangiopathic retinopathy in experimental diabetic monkeys. Trans Am Ophthalmol Soc 1988;86:389-421.

5 Kozak I, et al: Hyperreflective sign in resolved cotton wool spots using high-resolution optical coherence tomography and optical coherence tomography ophthalmoscopy. Ophthalmology 2007;114:537-543.

6 Matas BR: The optic fundus and hypertension. Med Clin North Am 1977;61:547-564.

7 Ioannides A, et al: Isolated cotton-wool spots of unknown etiology: management and sequential spectral domain optical coherence tomography documentation. Clin Ophthalmol 2011;5:1431-1433.

8 Kim JS, et al: The microperimetry of resolved cotton-wool spots in eyes of patients with hypertension and diabetes mellitus. Arch Ophthalmol 2011;129:879-884
9 Gomez ML, et al: Imaging of long-term retinal damage after resolved cotton wool spots. Ophthalmology 2009;116:2407-2414.

10 Jampol LM, Rabb MF: Vasoocclusive diseases of the posterior pole. Int Ophthalmol Clin 1981;21:201-213.

11 Bock KD: Regression of retinal vascular changes by antihypertensive therapy. Hypertension 1984;6:III158-III162.

12 Kohner EM, Dollery CT, Bulpitt CJ: Cottonwool spots in diabetic retinopathy. Diabetes 1969;18:691-704.

13 Mansour AM, et al: Cotton-wool spots in acquired immunodeficiency syndrome compared with diabetes mellitus, systemic hypertension, and central retinal vein occlusion. Arch Ophthalmol 1988;106:1074-1077.

14 Sacu S, et al: Response of retinal vessels and retrobulbar hemodynamics to intravitreal anti-VEGF treatment in eyes with branch retinal vein occlusion. Invest Ophthalmol Vis Sci 2011;52:3046-3050.

15 Munk MR, Kiss CG, Huf W, Montuoro A, Sulzbacher F, Kroh M, Larsen M, SchmidtErfurth U: Visual acuity and microperimetric mapping of lesion area in eyes with inflammatory cystoid macular oedema. Acta Ophthalmol 2014;92:332-338.

16 Munk M, et al: Detection and differentiation of intraretinal hemorrhage in spectral domain optical coherence tomography. Curr Eye Res 2014;20:1-9.

17 Archer DB: Tributary vein obstruction: pathogenesis and treatment of sequelae. Doc Ophthalmol 1976;40:339-360.

18 Mansour AM, Rodenko G, Dutt R: Half-life of cotton-wool spots in the acquired immunodeficiency syndrome. Int J STD AIDS 1990;1: 132-133.

19 Fawzi AA, et al: Acute macular neuroretinopathy: long-term insights revealed by multimodal imaging. Retina 2012;32:15001513.

20 Sarraf D, et al: Paracentral acute middle maculopathy: a new variant of acute macular neuroretinopathy associated with retinal capillary ischemia. JAMA Ophthalmol 2013;131: 1275-1287.

$21 \mathrm{Yu}$ S, et al: Multimodal imaging findings in retinal deep capillary ischemia. Retina 2014; 34:636-646.

22 Munk MR, Mirza RG, Jampol LM: Imaging of a cilioretinal artery embolisation. Int J Mol Sci 2014;15:15734-41570.

23 Hodge JV, Dollery CT: Retinal soft exudates. a clinical study by colour and fluorescence photography. Q J Med 1964;33:117-131.

24 Dollery CT, et al: I. Ophthalmoscopic and circulatory changes in focal retinal ischaemia. $\mathrm{Br}$ J Ophthalmol 1966;50:285-324. 